\title{
Malaria Treatment Policy Change and Implementation: The Case of Uganda
}

\author{
Miriam Nanyunja, ${ }^{1}$ Juliet Nabyonga Orem, ${ }^{2}$ Frederick Kato, ${ }^{3}$ Mugagga Kaggwa, ${ }^{1}$ \\ Charles Katureebe, ${ }^{1}$ and Joaquim Saweka ${ }^{4}$ \\ ${ }^{1}$ Disease Prevention and Control Cluster: World Health Organization, Uganda Country Office, P.O. Box 24578, Kampala, Uganda \\ ${ }^{2}$ Health Systems and Services Cluster: World Health Organization, Uganda Country Office, P.O. Box 24578, Kampala, Uganda \\ ${ }^{3}$ National Malaria Control Program: Ministry of Health P.O. Box 7272, Kampala, Uganda \\ ${ }^{4}$ World Health Organization, Uganda Country Office, P.O. Box 24578, Kampala, Uganda
}

Correspondence should be addressed to Juliet Nabyonga Orem, nabyongaj@ug.afro.who.int

Received 17 March 2011; Accepted 7 July 2011

Academic Editor: Ananias Escalante

Copyright ( $) 2011$ Miriam Nanyunja et al. This is an open access article distributed under the Creative Commons Attribution License, which permits unrestricted use, distribution, and reproduction in any medium, provided the original work is properly cited.

Malaria due to P. falciparum is the number one cause of morbidity and mortality in Uganda where it is highly endemic in $95 \%$ of the country. The use of efficacious and effective antimalarial medicines is one of the key strategies for malaria control. Until 2000, Chloroquine (CQ) was the first-line drug for treatment of uncomplicated malaria in Uganda. Due to progressive resistance to CQ and to a combination of CQ with Sulfadoxine-Pyrimethamine, Uganda in 2004 adopted the use of ACTs as first-line drug for treating uncomplicated malaria. A review of the drug policy change process and postimplementation reports highlight the importance of managing the policy change process, generating evidence for policy decisions and availability of adequate and predictable funding for effective policy roll-out. These and other lessons learnt can be used to guide countries that are considering anti-malarial drug change in future.

\section{Introduction}

Malaria is the number one cause of morbidity and mortality in Uganda. It is highly endemic in $95 \%$ of the country, and the remaining $5 \%$ of the country is prone to malaria epidemics [1]. Over 95\% of the malaria cases are due to plasmodium falciparum. Effective malaria case management, using efficacious and effective antimalarial medicines, is one of the recommended strategies for malaria control [2]. Until 2000, Chloroquine (CQ) was the first-line medicine for treatment of uncomplicated malaria in Uganda, and Sulfadoxine/Pyrimethamine (SP) or Amodiaquine (AQ) was the 2nd-line medicine while Quinine $(\mathrm{Qn})$ was the reserve medicine. For severe malaria, Qn was the recommended medicine initially given intravenously until the patient is conscious and able to take medicines orally [3]. However, in the late 1990s, parasite resistance to CQ, at varying levels in the 8 East African Network for Monitoring Antimalarial Treatment (EANMAT) sentinel sites located in different parts of the country, was documented. By 2000, the parasitological resistance to CQ had increased significantly, ranging from $<5 \%$ to $>50 \%$ in different sites, and clinical failure following CQ treatment in Uganda had increased to about 38\% [4], exceeding the WHO recommended threshold of clinical failure of $25 \%$, beyond which policy change is recommended in the shortest time possible [5]. Uganda embarked on a malaria treatment policy change process as shown in Figure 1. After several technical discussions and considerations, the firstline antimalarial medicine for uncomplicated malaria was changed from CQ only, and a new interim policy with a combination of CQ and Sulfadoxine-Pyrimethamine (CQ/SP) as first-line treatment was adopted. The selection of CQ/SP was based on assumption that the combination would be more efficacious than CQ monotherapy [5]. In addition, the government could ensure availability of the CQ/SP combination to all public health facilities using available resources. However, between 2001 and 2004, the efficacy of $\mathrm{CQ} / \mathrm{SP}$ reduced significantly, with treatment failure ranging 


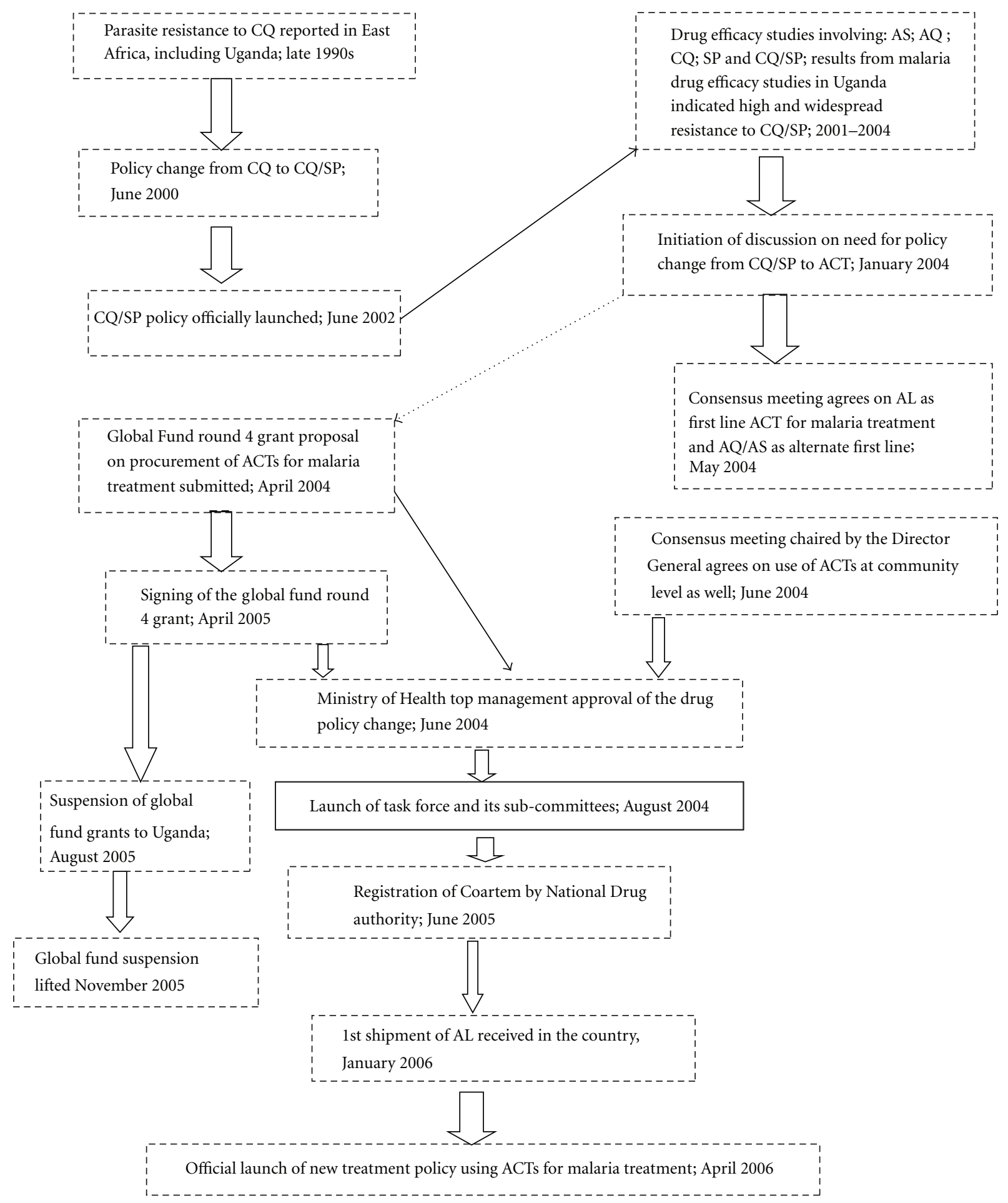

FIGURE 1: A timeline of key events during the malaria drug policy change in Uganda.

from $22 \%$ to $77 \%$ (median $41 \%$ ) [6-9]. In line with the WHO recommended threshold for antimalarial drug policy change (clinical failure rate of $\geq 25 \%$ ), Uganda embarked on yet another policy change process that culminated in the adoption of artimesinin combination therapies (ACTs) as the first-line treatment for uncomplicated malaria. Quinine was selected as the second-line treatment for uncomplicated malaria and first-line treatment for severe malaria and for malaria in pregnancy. Specifically, Artemether-Lumefantrine (AL) was adopted as the first-line treatment for uncomplicated malaria, with Artesunate-Amodiaquine (AS/AQ) as an alternative first-line [10]. This paper describes this last policy change process, factors put into consideration prior to adoption of this policy, the roll-out of the policy, 5-year 
experience of implementation of the new policy, and the lessons learnt, to guide other countries that consider malaria treatment policy change.

\section{Methods}

We reviewed unpublished reports and scientific publications on several drug efficacy studies conducted in Uganda between 2000 and 2004 to document the evidence used in selection of the first-line antimalarial drug. We also reviewed unpublished reports and minutes of several meetings that were held to gain consensus on the medicine to adopt as first-line for treatment of uncomplicated malaria, the reports of the task force that was constituted to guide the implementation of the new policy and its subcommittees, the minutes of the different meetings held by the subcommittees and the task force, as well as the documentation of the malaria treatment policy change process that summarized the whole process step by step and included some of the key reports as attachments. For experiences on the implementation of the policy, and challenges faced, we reviewed several reports and publications by different groups of people that conducted studies to assess the level of implementation of the new policy. The studies were done at different periods after the introduction of the policy. We also used information from National Medical Stores (NMS) and National Malaria Control Programme (NMCP) reports, the Malaria Indicator Survey report, and Annual Health Sector Reports for the details on policy implementation and drug availability. However, and most important, the first and third authors participated in the whole policy change process, compiled the documentation of the drug policy change process, and thus also input personal experiences into the paper.

\subsection{Malaria Treatment Policy Change Process}

2.1.1. Decision-Making Process. In 2004, results of malaria drug efficacy studies in Uganda showing high and widespread resistance to CQ/SP (Table 1) caught the Ministry of Health $(\mathrm{MoH})$ by surprise as it had not been adequately updated on the growing resistance by the researchers. This happened around the same time that WHO released the WHO statement on ACTs as the most effective antimalarials that should be considered as first-line treatment for malaria in highly endemic countries with CQ resistance [11]. At the same time there was international pressure and advocacy from malaria experts about the need to change malaria treatment policy to ACTs as first-line treatment in several countries including Uganda [12]. Several technical meetings were held by partners including WHO, Malaria Consortium, and the researchers with $\mathrm{MoH}$, until the need to change malaria treatment policy was appreciated, accepted, and commissioned by the MoH. Policy change discussions were then initiated, with support from WHO and other partners.

Whereas in principle policy change to ACTs was inevitable, the cost of ACTs was prohibitive and unaffordable by the Government of Uganda. However, there were prospects of funding for ACTs by the Global Fund for AIDS,
Tuberculosis, and Malaria (GFATM). Uganda responded to a call for round 4 proposals by the GFATM and submitted a 5-year grant proposal to support the procurement and introduction of ACTs for malaria treatment in Uganda. The success of the round 4 GFATM proposal and hence assurance of funds for ACTs for 5 years paved way for discussions to consider adoption of ACTs in Uganda.

A one-day technical meeting was jointly convened by $\mathrm{WHO}$ and $\mathrm{MoH}$ to review the evidence available and make a decision on which ACT to adopt. The meeting was attended by technical experts in malaria case management in Uganda, Malaria researchers from Makerere University and University of California, San Francisco collaboration, technical advisors from WHO headquarters, Regional Office for Africa, and Country office, Malaria consortium, USAID, DFID, and MSF. Results of the various studies on efficacy of $\mathrm{CQ} / \mathrm{SP}$, other non-ACT medicines, and ACTs (Table 1) and one study on effectiveness of Artemether-Lumefantrine (AL) [13] were presented and discussed. WHO technical experts also made a presentation on WHO guidance and its basis as well as the recommended ACTs [11]. Discussions held on which ACTs to adopt covered efficacy and effectiveness, drug formulation with a preference for coformulated tablets to copackaged ones, availability of a pediatric formulation, ease of administration, possible adverse effects, cost, and ease of use by both qualified health workers and community health workers during home-based management of fever (Table 2). Though ACTs were expensive, with Artemether/ Lumefantrine (AL) being more expensive than Amodiaquine and Artesunate $(\mathrm{AQ} / \mathrm{AS})$, it was anticipated that, in the future, prices would come down due to economies of scale. Besides, funding had already been secured from the GFATM for procuring adequate quantities of ACTs for the foreseeable future. Discussions were tough, with different groups having different ACT preferences due to personal and possible external influences, but consensus was achieved; AL was selected as the first-line antimalarial. After further review by the $\mathrm{MoH}$ technical team, AQ/AS was selected as an alternative first-line drug (Table 2). Agreement on what drug to adopt for home-based management of fever was harder, and consensus was not achieved at this meeting, with some experts suggesting a combination of Amodiaquine and $\mathrm{SP}(\mathrm{AQ} / \mathrm{SP})$ that was still fairly efficacious instead of taking ACTs to community level. The Director General of Health Services convened another technical meeting to discuss this further, and finally it was agreed that first-line ACTs should be accessed by all people with uncomplicated malaria at both health facility and community level.

Parasite resistance to CQ/SP was above the WHO treatment failure threshold in some regions and was reported to be below the threshold in other regions. This raised questions on whether the policy change should be applied to some parts of the country and leave the others using CQ/SP until the treatment failure rate reaches the threshold for drug policy change. After review of the logistical challenges of implementing a dual first-line policy, it was agreed that the policy would be changed for the whole country. A brief about the proposed change in malaria treatment policy was written and presented to the $\mathrm{MoH}$ top management for approval by 
TABLe 1: Antimalarial drug efficacy studies in Uganda from 2000 to 2001: results for children less than 5 years.

\begin{tabular}{|c|c|c|c|c|c|c|c|}
\hline \multirow{2}{*}{$\begin{array}{l}\text { Period of } \\
\text { study }\end{array}$} & \multirow{2}{*}{$\begin{array}{l}\text { Site, transmission } \\
\text { intensity }\end{array}$} & \multirow{2}{*}{$\begin{array}{l}\text { Researchers/authors } \\
\text { and/or publication }\end{array}$} & \multirow{2}{*}{ Protocol used } & \multirow{2}{*}{$\begin{array}{l}\text { Drug or drug } \\
\text { combination }\end{array}$} & \multicolumn{2}{|c|}{ Treatment failure rates (\%) } & \multirow{2}{*}{$\begin{array}{r}\text { Parasitological } \\
\text { failure rate }(\%)\end{array}$} \\
\hline & & & & & After 14 days & After 28 days & \\
\hline \multirow{3}{*}{2000} & \multirow{3}{*}{ Kampala, Medium } & \multirow{3}{*}[14]{} & \multirow{3}{*}{ WHO 1996} & $\mathrm{SP}$ & 10 & - & - \\
\hline & & & & AQ & 7 & - & - \\
\hline & & & & $\mathrm{AQ} / \mathrm{SP}$ & 3 & - & - \\
\hline \multirow{3}{*}{$\begin{array}{l}\text { July } 2000 \text { to } \\
\text { August } 2001\end{array}$} & \multirow{3}{*}{ Kampala, Medium } & \multirow{3}{*}[15]{} & \multirow{3}{*}{$\begin{array}{l}\text { Longitudinal } \\
\text { study }\end{array}$} & $\mathrm{SP}$ & 17 & - & 32 \\
\hline & & & & $\mathrm{AQ} / \mathrm{SP}$ & 1 & - & 2 \\
\hline & & & & AS/SP & 1 & - & 5 \\
\hline \multirow{3}{*}{$\begin{array}{l}\text { July to Sept } \\
2001\end{array}$} & \multirow{3}{*}{ Kaberamaido, high } & \multirow{3}{*}[16]{} & \multirow{3}{*}{ WHO 1996} & CQ & - & 45 & - \\
\hline & & & & SP & - & 16 & - \\
\hline & & & & $\mathrm{CQ} / \mathrm{SP}$ & - & 12 & - \\
\hline \multirow{3}{*}{ October 2001} & \multirow{3}{*}{ Kabale, low } & & & CQ & 7.5 & - & - \\
\hline & & {$[17]^{*}$} & WHO 1996b & SP & 0 & - & - \\
\hline & & & & $\mathrm{CQ} / \mathrm{SP}$ & 0 & - & - \\
\hline & & & & $\mathrm{SP}$ & - & 15 & 30 \\
\hline $\begin{array}{l}\text { March } 2001 \\
\text { to Jan } 2002\end{array}$ & Kampala, Medium & {$[18]^{*}$} & WHO 1996 & $\mathrm{CQ} / \mathrm{SP}$ & - & 7 & 17 \\
\hline & & & & $\mathrm{AQ} / \mathrm{SP}$ & - & 0 & 1 \\
\hline & & & & CQ/SP & 8 & - & 40 \\
\hline March 2002 & Tororo, high & {$[7]$} & WHO 1996 & $\mathrm{AQ} / \mathrm{SP}$ & 0 & - & 15 \\
\hline & & & & $\mathrm{SP}$ & 9 & - & 42 \\
\hline & & & & SP & 23.4 & 37 & - \\
\hline $\begin{array}{l}\text { Jan to Nov } \\
2002\end{array}$ & Bundibugyo, high & {$[19]$} & WHO 2002 & $\mathrm{AQ}$ & 8.8 & 20.6 & - \\
\hline & & & & $\mathrm{CQ} / \mathrm{SP}$ & 6.0 & 22.8 & - \\
\hline & & & & $\mathrm{CQ} / \mathrm{SP}$ & - & 35 & - \\
\hline $\begin{array}{l}\text { Aug } 2002 \text { to } \\
\text { July } 2003\end{array}$ & $\begin{array}{l}\text { Mulago Hospital, } \\
\text { Kampala Medium }\end{array}$ & {$[9]$} & WHO 1996 & $\mathrm{AQ} / \mathrm{SP}$ & - & 9 & - \\
\hline & & & & AQ/AS & - & 2 & - \\
\hline & Kanungu, low & & WHO 1996 & $\mathrm{CQ} / \mathrm{SP}$ & - & 67 & 73 \\
\hline & Nanumgu, low & {$[8]$} & VV10 1990 & $\mathrm{AQ} / \mathrm{SP}$ & - & 35 & 38 \\
\hline & Kyenjojo, high & & WHO 1996 & $\mathrm{CQ} / \mathrm{SP}$ & - & 37 & 58 \\
\hline Dec 2002 to & куепро, ngn & {$[8]$} & VमПU 1990 & $\mathrm{AQ} / \mathrm{SP}$ & - & 14 & 24 \\
\hline June 2003 & Mubende, medium & & WHO 1996 & $\mathrm{CQ} / \mathrm{SP}$ & - & 34 & 43 \\
\hline & & {$[8]$} & & $\mathrm{AQ} / \mathrm{SP}$ & - & 13 & 14 \\
\hline & All sites combined & & WHO 1996 & $\mathrm{CQ} / \mathrm{SP}$ & 22 & Range 34-67 & Range 43-73 \\
\hline & All sites comomed & {$[8]$} & WП10 1990 & $\mathrm{AQ} / \mathrm{SP}$ & 8 & Range 13-35 & Range 14-38 \\
\hline & & & & CQ/SP & - & 40 & - \\
\hline & $\begin{array}{l}\text { Jinja, low to } \\
\text { medium }\end{array}$ & {$[20]$} & WHO 2003 & AQ/SP & - & 13 & - \\
\hline & & & & AQ/AS & - & 4 & - \\
\hline & Arua, medium to & & & $\mathrm{CQ} / \mathrm{SP}$ & - & 46 & - \\
\hline & $\begin{array}{l}\text { Arua, medium to } \\
\text { high }\end{array}$ & {$[20]$} & WHO 2003 & $\mathrm{AQ} / \mathrm{SP}$ & - & 14 & - \\
\hline & & & & $\mathrm{AQ} / \mathrm{AS}$ & - & 9 & - \\
\hline Nov 2002 to & & & & $\mathrm{CQ} / \mathrm{SP}$ & - & 34 & - \\
\hline May 2004 & Tororo, high & {$[20]$} & WHO 2003 & $\mathrm{AQ} / \mathrm{SP}$ & - & 18 & - \\
\hline & & & & $\mathrm{AQ} / \mathrm{AS}$ & - & 12 & - \\
\hline & & & & $\mathrm{CQ} / \mathrm{SP}$ & - & 22 & - \\
\hline & Apac, high & {$[20]$} & WHO 2003 & $\mathrm{AQ} / \mathrm{SP}$ & - & 7 & - \\
\hline & & & & $\mathrm{AQ} / \mathrm{AS}$ & - & 10 & - \\
\hline & All sites combined & {$[20]$} & WHO 2003 & $\mathrm{CQ} / \mathrm{SP}$ & - & $22-46$ & - \\
\hline & & & & $\mathrm{AQ} / \mathrm{SP}$ & - & $7-18$ & - \\
\hline & & & & AQ/AS & - & $4-10$ & \\
\hline
\end{tabular}

* Study participants included both children and adults; results reported for both children and adults together. AS: Artesunate; AQ: Amodiaquine, CQ: Chloroquine, SP: Sulfadoxine-Pyrimethamine. 
TABLE 2: How the decision to select the first-line treatment was reached.

\begin{tabular}{|c|c|c|}
\hline Combination & Decision & Comments \\
\hline Artemether/Lumefantrine (3 days-course) & Possible & $\begin{array}{l}\text { Shown good efficacy in Uganda, Kenya, Tanzania and other } \\
\text { African countries; good effectiveness in Uganda; } \\
\text { coformulated tablets, prepacked in treatment courses for } \\
\text { specific age groups }\end{array}$ \\
\hline Artesunate ( 3 days $)+$ Amodiaquine (3 days) & Possible & $\begin{array}{l}\text { Shown good efficacy in Uganda; co-packaged; provider and } \\
\text { consumer acceptance of Amodiaquine was low hence } \\
\text { co-packaging instead of coformulation could encourage } \\
\text { administering Artesunate and leaving Amodiaquine, some } \\
\text { resistance to the combination already documented in Uganda }\end{array}$ \\
\hline $\begin{array}{l}\text { Artesunate ( } 3 \text { days })+ \\
\text { Sulfadoxine/Pyrimethamine ( } 1 \text { day) }\end{array}$ & Rejected & $\begin{array}{l}\text { There was already high resistance of } P \text {. falciparum to SP in } \\
\text { Uganda and in the sub-region; and already some resistance to } \\
\text { Artesunate-SP combination in Uganda }\end{array}$ \\
\hline $\begin{array}{l}\text { Amodiaquine ( } 3 \text { days })+ \\
\text { Sulfadoxine/Pyrimethamine ( } 1 \text { day) }\end{array}$ & Rejected & $\begin{array}{l}\text { According to WHO recommendations this combination } \\
\text { should be an interim policy recommended where ACTs } \\
\text { cannot be immediately deployed and where parasite } \\
\text { resistance to both AQ and SP is still low. Already in Uganda } \\
\text { there was high resistance of } P \text {. falciparum to SP. There was } \\
\text { also high probability of cross-resistance between AQ and CQ, } \\
\text { reducing further the efficacy of AQ+SP combination. Already } \\
\text { one site (Tororo) showed parasitological resistance to AQ+SP } \\
\text { combination of } 32.5 \%\end{array}$ \\
\hline
\end{tabular}

Artesunate (3 days) + Mefloquine (MQ) $\mathrm{AS} / \mathrm{MQ}$

Not recommended by WHO for high transmission areas.

the NMCP. After a long discussion on financial, logistical, mortality reduction and other implications of the policy change, the top $\mathrm{MoH}$ managers accepted the proposed policy change in June 2004. This paved the way for the planning for the launch and implementation of the policy change. The new policy was officially launched by the Rt. Honourable Prime Minister of Uganda, on behalf of His Excellency the President of Uganda, on the 25th of April 2006 during the commemoration of Africa Malaria Day.

2.1.2. Planning for Policy Change. A task force was established by Ministry of Health to guide the planning for the rolling out of the policy change. Four subcommittees of the task force, commissioned in August 2004, were tasked with development of different aspects of the roll-out plan. The treatment guidelines and training approaches subcommittee updated the malaria treatment guidelines and job aids to include the new policy details and also developed other training materials and the training plan for health workers and community medicine distributors; the Information, Education, and Communication (IEC), advocacy and social mobilization subcommittee updated the IEC materials, developed a training module for health workers and a handbook for parliamentarians as well as the overall communication strategy to support the policy change; the supply management subcommittee quantified the ACTs needed annually using the morbidity method, proposed mechanisms for incorporating AL into the Essential Medicines List for Uganda and for phasing out CQ/SP and phasing in ACTs while the monitoring and evaluation subcommittee worked on monitoring framework for the policy roll-out, covering process, output, and impact monitoring, incorporating $\mathrm{AL}$ into the existing antimalarial medicines efficacy studies as well as establishment of pharmacovigilance and postmarketing surveillance. A meeting was held at central level in September 2004, where the products of the different subcommittees were discussed, agreed upon and a team tasked to compile these into one-policy implementation plan. Relevant IEC and training materials were ready for mass production for the policy implementation. Introduction of ACTs was planned for in phased manner, starting with the health facilities in 2005/2006 and to extend to communities for home-based management of fever at beginning of 2007.

2.1.3. Registration of ACTs. AL (Coartem) was officially registered as an antimalarial medicine by the Uganda National Drug Authority (NDA) in June 2005. It was initially classified as a prescription-only medicine although steps were taken later to reclassify it to an over-the-counter medicine to enable its use at community level for the home-based management of fever. The public-sector Coartem was in form of 4 colourcoded packs: yellow with 6 tablets per pack for the treatment of people with weight ranging from 5 to $14 \mathrm{~kg}$, blue with 12 tablets per pack, for the $15-24 \mathrm{~kg}$ group, brown with 18 tablets per pack, for the $25-34 \mathrm{~kg}$ group, and green with 24 tablets per pack, for the $\geq 35 \mathrm{~kg}$ weight group. In Uganda, to ease prescription, case management experts translated the weights into matching age groups with yellow Coartem for treatment of people from 4 months to 3 years, blue for 4 to 7 years, brown for 8 to 12 years, and green for those above 12 years. Coartem for private-sector use was packaged differently. Several other brands of AL besides Coartem that were subsequently registered by the NDA include Lumartem and Lumet Forte of CIPLA LTD. and Artefan of AJANTA 
Pharmaceuticals. Other ACTs that were also later registered in their various brands included Artesunate + Amodiaquine, Artesunate + SP, and Dihydroartemisinin + Piperaquine [21]. Availability of several ACTs ensured a variety of ACTs in the private sector. Ensuring use of high-quality ACTs in the private sector is however still a challenge; a study done in 2010 in 3 African countries including Uganda found substandard ACTs on the market. In the case of Uganda, they were only in the private sector [22].

To avoid use of artimesinin monotherapies that could result in quick development of parasite resistance to artimesinin and ACTs in general, the government (NDA) banned importation of oral artimesinin monotherapies in 2007 , although implementation may not be $100 \%$ enforced. This resulted in significant decline of oral artimesinin monotherapies in the private sector.

\subsection{Artimesinin Combination Therapy Introduction Process}

2.2.1. Funding for ACT Introduction Process. GFATM round 4 that was the source of funding for the policy change was signed in April 2005, and first fund disbursement was in December 2005. Following the disbursement from GFATM, orders for ACTs were made by the country, through an arrangement with WHO; WHO had an agreement with Novartis, the manufacturer to AL (Coartem) that ensured that countries got the drug at a very subsidized cost for the public sector. Funds for preparatory activities including mass production of training materials, job aides, malaria treatment guidelines, and different IEC materials were also disbursed and activities initiated in January 2006. Whereas the policy change was accepted in June 2004, it was not until April 2006 that actual implementation started. This time lag was mainly due to delays in GFATM grant signing and release of funds to the country and from the government treasury to the $\mathrm{MoH}$.

2.2.2. Training. Cascade training was implemented, starting with training of national-level trainers who trained district trainers. Training at both levels was in form of facilitated workshops. Samples of the prepackaged and color-coded AL were available at these trainings for trainees to familiarize with. The district trainers conducted on-job training of the health workers and familiarized health workers with the samples of the drug packs. Training was followed immediately by deployment of the medicines, to ensure that health workers immediately initiate ACT prescription as trained, prior to knowledge decay. The health workers in the private sector were also trained; however private practitioners had to procure ACTs on the open market, where it was about 10 times the cost of the public-sector ACTs. This eventually encouraged pilferage of the public sector ACTs to some private facilities, a practice that was actively investigated and condemned by the government authorities.

2.2.3. Social Mobilization. A national launch of the new treatment policy was done by the Rt. Hon. Prime Minister, on the 25th of April 2006, on behalf of His Excellency the
President of Uganda. IEC campaign about the new and more effective antimalarial drug was conducted using radios, television, posters, among others. Interpersonal communication was also used in sensitization meetings with district leaders, health workers, parliamentarians, and different groups at community levels. The message reached most communities and increased the demand for the new drug by patients at the health facilities. One of the key IEC materials for health workers in public and private sector was a one-page chart with the summarized new drug policy in tabular form, which they hang in all out-patient consultation rooms and inpatient wards for continuous reference. The campaign was generally successful and informative.

2.2.4. Logistics Management. ACTs were procured through WHO arrangement with Novartis for the first 3 years of implementation, in line with the procurement plan developed in planning phase. In quantification and ordering for the medicines, data from a sample of health facilities were used to ascertain the proportions of AL of the different colors needed. The first shipment of AL was received on the 31st of January 2006. Handling and distribution of ACTs were from the onset mainstreamed into the existing government process of clearing, storage, and distribution of medicines by the National Medical Stores (NMS) for public sector and Joint Medical Stores (JMS) for private not-for-profit (PNFP) sector. The NMS had to hire additional storage space to store the AL prior to distribution as the drug was more bulky than CQ/SP. JMS would receive from NMS supplies for the PNFP health facilities (about 20\% of the medicines) and was in position to handle storage and distribution. The first distribution of AL was a "push" to all health facilities based on morbidity estimates made at national level based on Health Management and Information System (HMIS) data; subsequently orders were made by the health facilities based on estimated needs, also using the morbidity method. As this was a new drug and health facilities were not very clear as to the proportion of people in the different agegroups attending the health facility, there were overestimates and underestimates of required AL of different colors by different districts and health facilities. Due to this, often the total orders received from the districts were exceeding the procured monthly AL. Hence, some rationing was done at the NMS to ensure that all health facilities received some ACTs. Maintaining adequate stocks of the different color codes was a challenge at both national and health facility levels. Adherence to the NMS delivery schedules was also a challenge due to the bulkiness of the drug, the old transport fleet then, and management challenges within the institution. Delayed delivery of AL to the district and health facilities contributed to stock-out at these levels.

2.2.5. Monitoring. To monitor the implementation of the new malaria treatment policy, troubleshoot where problems are being faced and ensure use of CQ/SP and monotherapies is phased out, quarterly support supervision was planned for in the first year. However, the planned frequency of support supervision was not achieved due to late release of funds and 
other competing activities that were being undertaken by the national and district trainers. Support supervision was hence done in samples of districts after 6 to 9 months after the introduction of the new policy. The concept of pharmacovigilance and the forms for reporting any adverse effects of the drug were introduced to the health workers during the training and the reporting channel to the NDA clarified. HMIS tools were revised to capture ACTs stock-outs instead of CQ/SP, malaria cases and deaths, and malaria in pregnancy as a separate variable during the HMIS review of 2005. The updated forms were disseminated to all districts for use in reporting with effect from 2006. HMIS data was received monthly from all districts by the National Resource Center at the Ministry of Health and entered in the National Data Bank. The malaria specific information was retrieved from the data bank and reviewed periodically by the NMCP.

2.2.6. Technical Assistance. To support the introduction of the new policy, technical assistance was provided to the Ministry of Health by the WHO Country Office, the MSH, and Malaria Consortium. In addition to technical experts within these organizations, a full-time technical consultant attached to the NMCP was supported by WHO to assist the programme for 6 months in coordination of the different planned activities during policy change introduction process. In addition, a technical assistant was hired by MSH to support NMS in ACT-policy-related logistics management. All these technical experts assisted the Ministry of Health to ensure that all necessary steps as per WHO guidelines for policy change were undertaken and also timely troubleshooting about obstacles done.

\subsection{Implementation of the New Policy}

2.3.1. Review of Implementation of the New Policy. Details of evaluation studies undertaken to assess implementation of the new malaria treatment policy are summarized in Table 3. Three 3 months following the introduction of the new medicines/malaria treatment policy, as part of the documentation of the malaria treatment policy change, a survey was done in 5 districts ( 15 health facilities). The study found that guidelines on the new policy were available in study districts except the malaria treatment brochure which was not available in any of the districts. Overall, in all the 5 districts, AL was prescribed for $32 \%$ of uncomplicated malaria cases (range 5-72\%), even when AL was available at the health facility. AL stock-outs were reported as rampant in most of the districts [23]. Another study was done 11 months following introduction of the new malaria treatment policy covering 7 districts (119 health facilities), and stock-outs of $\mathrm{AL}$ were again registered. The study further showed that even the second-line drug, Qn, was out of stock in a significant number of health facilities. Seven (7) facilities lacked both AL and Qn for the three months prior to the survey with a danger of health workers prescribing other ineffective non-ACT antimalarials [24]. Another follow-up study done 11 months following the malaria treatment policy change showed that only $56 \%$ of uncomplicated malaria cases were prescribed
AL, even when the drug was available, again with significant stock-outs. Guidelines on malaria treatment policy change were only available in $50 \%$ of health facilities [25]. Another study undertaken 16 months following the malaria treatment policy change indicated that AL was prescribed for $64 \%$ of uncomplicated malaria, the rest were given $\mathrm{CQ} / \mathrm{SP}$ or any other non-ACT antimalarial. In some facilities that still had a lot of stock of CQ/SP, ACTs were being used alongside the CQ/SP with the clinician left to make the judgment on whom to give ACTs or CQ/SP. The tendency was for ACTs to be prescribed for more ill-looking patients and those who had failed to improve on CQ/SP. Stock-outs of AL were still reported; there were still significant amounts of CQ and SP available on the contrary, and availability of the alternate first-line drug AQ/AS was very low, below 5\%. Stocks of non recommended antimalarials available on the day of the survey were much higher than what had been reported 6 months earlier. Supervision on proper use of AL was also noted to be weak with only $34 \%$ of health facilities reporting having been supervised prior to the survey [26]. Malaria wall charts, displaying the new treatment policy (1st line, alternate first line, 2nd line, and medicines for malaria in pregnancy) and their dosages in tabular and pictorial form, that were provided to health facilities to serve as a quick guide for health workers were only available in $48 \%$ of sampled facilities despite having been disseminated at the time of launching the new policy [26].

2.4. Funding Mechanisms and Their Effects on Policy Implementation. In August 2005, about a year after the approval of the new malaria treatment policy by the Ministry of Health, 4 months after the signing of GFATM grant round 4 phase 1 agreement, and before the procurement of the first year's ACTs, all GFATM grants to Uganda were suspended following claims of mismanagement [27]. As GFATM was the main source of funds for procurement of ACTs, implementation of the new policy had to be postponed pending lifting of the suspension. After some negotiations, funding of life-saving commodities like ACTs and ARVs was approved even during the suspension, hence the procurement of the first year's ACTs in 2006. Introduction of ACTs had been planned for in a phased manner, starting with the health facilities and to extend to communities for home-based management of fever by early 2007 . The production of the prepacked CQ/SP for home-based management of fever, branded Homapak, had been stopped in anticipation for the community roll-out of ACTs at the beginning of 2007. Due to the delays in signing the GFATM round 4 grant, its suspension shortly after the signing, and the delays in release of necessary funding, the community roll-out was postponed to 2008, yet no more Homapak was being produced. Hence, this channel of antimalarial access (through home-based management of fever at community level) was rendered nonfunctional hampering the efforts to increase accessibility to ACTs within 24 hours of fever onset. The suspension was lifted in November 2005. The procurement of the lifesaving commodities including ACTs, through WHO, during the suspension enabled the Ministry of Health to plan for the 


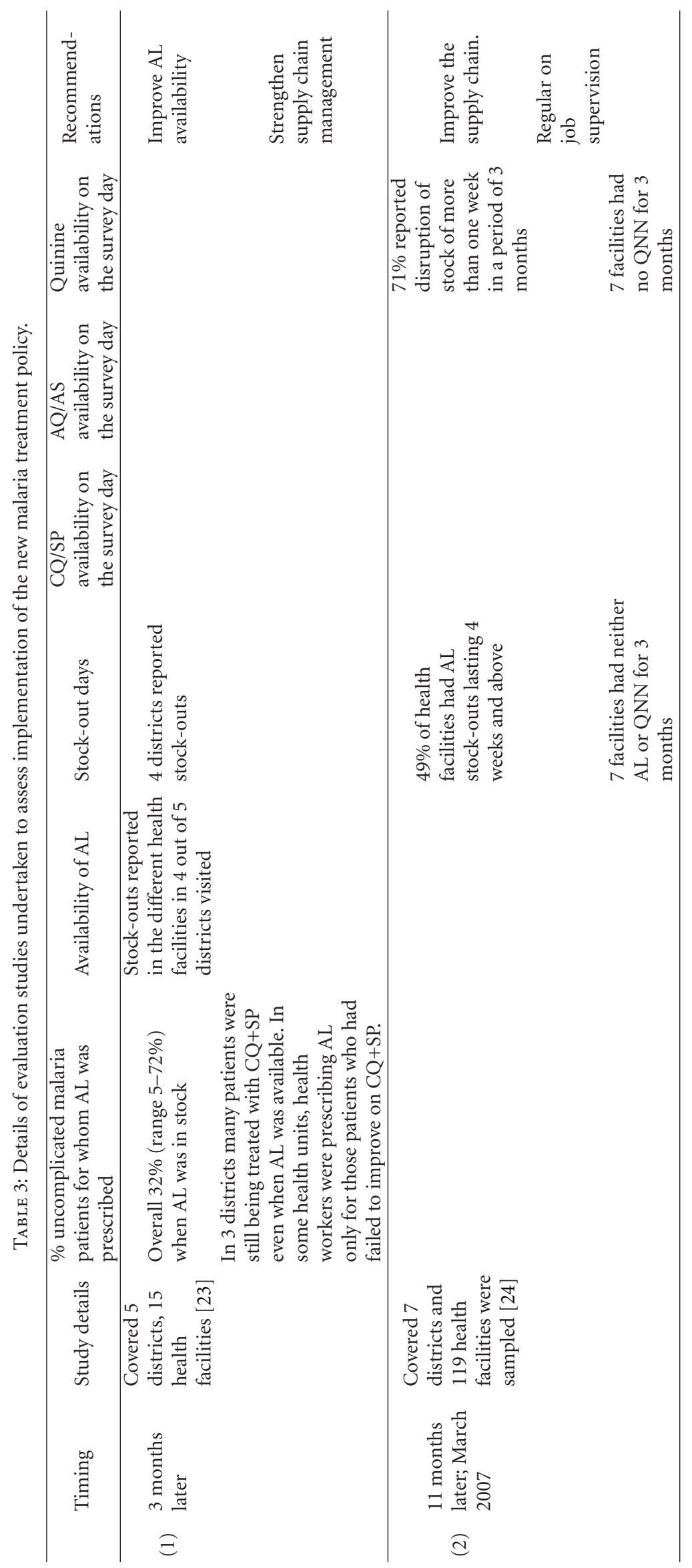




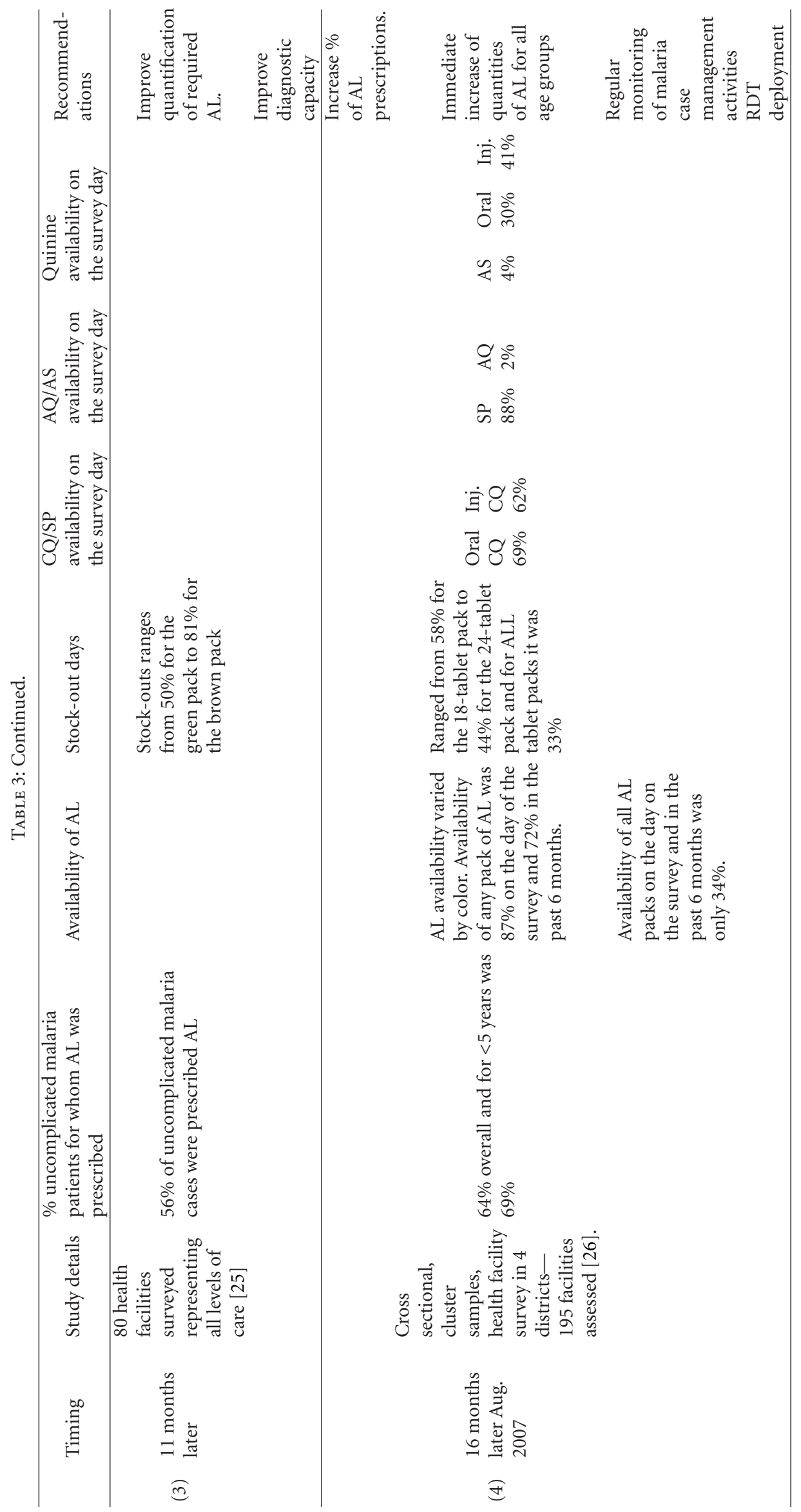


launch of the new malaria treatment policy in April 2006. After lifting the suspension, the disbursements were still not timely due to different reasons, thus resulting in stock-outs. The signing of GFATM round 4 grant phase 2 was delayed by almost 18 months during which there was again rampant stock-out of ACTs in the whole country.

2.5. Country Efforts to Address Drug Stock-Out Challenges. To increase local availability of ACTs and address the challenges of stock-outs, a local manufacturing plant, Quality Chemicals Industries Ltd., was established through collaboration between CIPLA and a Ugandan business group and commissioned in 2008. Soon after passing Good Manufacturing Practice (GMP), the factory started producing ACTs that were bought locally to try and fill in the gaps created by the delayed GFATM funds. However, this could not close the big gap and the demand from the community, especially during the 18 months of delay in signing of the GFATM round 4 grant phase 2 . Reasons for this were twofold; the production capacity of the local firm was low given the fact that it was in its infancy stage, and government expenditure on medicines was low yet it was supposed to purchase ACTs using own resources from the local firm. Per capita expenditure on medicines was only US\$ 1.7 in 2006/07 far below an estimated requirement of US\$ 7.5 [28]. During GFATM round 4 grant phase 2 period, Uganda was selected to pilot the Affordable Medicines Facility for malaria (AMFm) that would enable countries to access ACTs at a subsidized price, even for the private sector. There was a protracted negotiation between Government and AMFm as this seemingly good facility could potentially interfere with the prospects of the local manufacturing plant and country efforts to increase local availability of ACTs. However, as soon as a workable agreement was reached, Uganda accepted to be a pilot country for AMFm. Affordable Medicines Facility for malaria agreement was signed and launched on April 252011 (World Malaria Day). To date some of the first-line buyers in the private sector have already received about 2 million ACTs doses. This will hopefully contribute to more availability of ACTs and pave way for the eventual removal of CQ and SP for malaria treatment, leaving SP for only intermittent presumptive treatment of malaria in pregnancy.

\section{Discussion}

The experience of changing malaria treatment policy from CQ/SP to ACTs brings out several issues that are critical for consideration in policy change that constitute lessons learnt in the process. Several countries have gone through this process, and it is well appreciated that the malaria treatment policy change process is a complex endeavor and its successful implementation is influenced by several factors [29].

\section{Decision-Making}

Policy change for malaria treatment must be based on evidence of growing parasite resistance $[29,30]$. Although it is agreed that evidence to change policy should be of high quality and rigorous, the nature and amount of evidence required to change a drug policy can lead to protracted discussions [31]. In many countries evidence from efficacy studies has been used to justify malaria treatment policy changes [32] but it is now recognised that this alone is not sufficient [33]. Others have argued that given the complexities of changing drug polices the picture should be more comprehensive; evidence on cost effectiveness, cost of changing the drug policy, provider, and community acceptability and operational feasibility should be considered alongside efficacy data $[4,33]$. Indeed in Tanzania, after presentation of results from clinical trials showing parasite resistance to $\mathrm{CQ}$, further research was commissioned to generate evidence on cost effectiveness of alternative antimalarials and cost of changing the drug policy [34]. Other favorable factors include consistency of findings from several sites within the country, evidence drawn from a wide range of the study population, availability of local research with results similar to those in other countries, and credible partners like WHO being in agreement with presented evidence [30, 34]. In the case of Uganda, evidence presented was generated from several studies undertaken in different parts of the country, representing different levels of malaria endemicity. Results were consistent with those from Kenya, Tanzania, and Malawi. At around the same time, WHO released the position on ACTs as the most effective antimalarials [11], and international pressure from malaria experts about the need for a change in drug policy to ACTs as first-line treatment of malaria was also ongoing [12]. These factors were favorable to the dialogue on policy change in Uganda.

Several studies have emphasized the importance of institutionalizing malaria drug policy reviews based on systematically collected data, early and continuous engagement of all key stakeholders in the process, and improved communication $[34,35]$. In the case of Uganda, presentation of the data to the policy makers when resistance had already surpassed thresholds without having primed them of the growing resistance over time resulted in conspiracy suspicion. A multicountry analysis of the process of changing national malaria treatment policy showed that lack of standardized data, initial lack of understanding of how to use research findings to influence policy, and poor communication between key stakeholders were among the challenges faced [35].

In consideration of the data on efficacy of the different drug combinations, there is need to look at the current efficacy, the rate of deterioration of efficacy recorded in the country or in similar countries, the resistance mechanisms and possibility of cross-resistance and project how long the combination is likely to be of acceptable efficacy in the country if used well. If a fairly efficacious combination that has been deteriorating at a relatively fast rate is adopted, it is likely to exceed the policy change threshold and lead to another policy change process in a few years. This was the case with the policy change from CQ to CQ/SP. Whereas $\mathrm{AQ} / \mathrm{SP}$ had been proposed as an option for consideration for use in home-based management of fever, instead of taking ACTs to the community, the efficacy of the drug combination $\mathrm{AQ} / \mathrm{SP}$ had fallen significantly within a year [8] that it was not regrettable it had not been adopted as an option. 
What it costs to change and implement a policy has also been a challenge to taking optimal decisions especially in instances where the policy being adopted is more costly than the one in use [36-38]. In Uganda, the change from CQ to $\mathrm{CQ} / \mathrm{SP}$ was partly driven by the fact that this option was affordable to the government and patients seeking care in the private sector. Similarly in Tanzania, the change from $\mathrm{CQ}$ as a first-line drug to SP was driven by affordability despite the fact that research had shown a rising parasite resistance trend to SP [34]. Availability of the GFATM funds was a major influence on the adoption of the ACT treatment policy in Uganda. Although donor aid plays a key role in financing health services, the extent to which developing countries should make policy decisions based on funding from donors continues to be hotly debated. The lack of predictability, sustainability, and alignment has been documented in Uganda and other developing countries, hampering implementation of programmes [39-41]. Similarly this could hamper policy implementation. In the case of Uganda, the suspension of GFATM due to alleged mismanagement resulted in delayed implementation of the new policy, and the delay in signing the GFATM round 4 grant phase 2 resulted in rampant ACT stock-outs.

External influences on drug policy change can be several; donors through availing funds can influence drug policy change. Influence of donors in policy change processes in developing countries, largely tied on receiving donor aid, has been documented. In instances where this has been in conflict with local contexts, resistance from recipient countries has not always been successful [42]. Other external influences on policy change could be manufacturers and technical officers-leaning towards some manufacturers. In Uganda decision-making process, there was a lot of push for adoption of AS/AQ versus AL, with 2 different groups of people in the meeting pushing for the 2 medicines, which raised suspicion of the possibility of indirect influence by the manufacturers of the 2 drug combinations. The eventual adoption of $\mathrm{AL}$ as first-line and AS/AQ as alternative first-line was partly as a form of compromise. These influences were also documented in Tanzania where the change from CQ to SP was decampaigned by pharmaceuticals manufacturers and traders who had large stocks of CQ and had even made more investments to continue with CQ production and marketing [34]. As much as possible, manufacturers' influence should be avoided in the discussion on drug policy change and focus be put on efficacy, cost-effectiveness, acceptability, and feasibility of use of the different medicines. Provider and consumer confidence in the recommended drug policy is also critical for effective implementation. In Tanzania, a belief by the public that CQ was still effective despite results of efficacy studies that showed high parasite led to protracted discussions and loss of time in the policy change process [34]. The low provider and consumer acceptability of Amodiaquine due to the perceived severe side effects was an important factor in decision-making in Uganda. In addition, there were also concerns about co-packaging of AS/AQ against the background of low provider and consumer acceptability as this could result in situations where patients consume only Artesunate and not Amodiaquine and lack of pediatric for- mulation for AS/AQ at the time. However, the manufacturer has now started producing coformulated AS/AQ as well as the pediatric formulations.

\section{Social Mobilization}

Involvement of the public needs to be harnessed right from the time of undertaking the research, dissemination of results, to launching and implementing the policy. The public should know and understand why a new policy is being developed. Public suspicions, personal experiences, and lack of trust have often derailed policy change processes. In Tanzania, parliamentarians' public pronouncements that they were continuing to use CQ raised questions among the general public about the need to change the drug policy to SP. In addition, isolated incidences from patients who had had side effects from other antimalarials further detracted the debate [43]. Public pronouncements by high ranking official facilitates uptake of new polices. In Uganda, launch of the new treatment policy by the Rt. Hon. Prime Minister during the commemoration of Africa Malaria Day energized the policy implementation and reinforced government's commitment to fight malaria. In Tanzania, launch by the honorable minister of health also greatly enhanced public confidence [34]. However, caution also needs to be taken on what message is given and the potential effects of the message. They should be pretested well to ensure that they communicate the intended message. In Uganda, the message sent out that ACTs were the most effective drug had far reach but in some cases resulted in some unperceived actions. Some health workers would keep this very effective drug for the patients that were very sick looking or those who did not respond initially to CQ/SP. In the latter case, it was being used as a second line instead of first-line treatment.

The policy of clinical diagnosis and presumptive treatment of all fever, especially in children less than 5 years, as malaria [10], resulted in several non-malaria cases being treated as malaria. Whereas CQ had antipyretic effects and hence patients would initially feel a bit better on CQ even if they did not have malaria, AL lacked this quality. Hence febrile patients, misdiagnosed and treated as malaria, would in most cases not feel better while on AL, and this was quite disappointing to the public given the IEC messages passed. After some time, the public started discrediting AL, indicating that it is not as effective as had been portrayed. However, efficacy studies at the time still indicated that AL was quite efficacious. Subsequently, the Ministry of health adopted a malaria diagnostic policy that requires each febrile case to be subjected to parasitological diagnosis of malaria by microscopy or rapid diagnostic tests, and only parasitologically positive cases should be treated with antimalarials.

5.1. Cost. Treatment policy change is quite costly and should be well planned for. Although the highest cost falls on acquiring the drug, training, preparation of guidelines, communication, supervision, and monitoring of policy implementation, continued therapeutic efficacy testing of the drug and informing the policy makers when changes are needed 
are costly activities which are often underrated. In Tanzania, estimations showed that the cost of changing the malaria drug policy amounted to $1 \%$ of overall public expenditure and $3 \%$ of annual drug budget [44]. Key activities are often not undertaken compromising successful implementation of the policy. In Uganda, support supervision was not implemented as planned, and this possibly had a negative effect on the policy implementation.

Many of the malaria patients are attended to in the private sector. Hence, malaria treatment policy should be implemented by both the public and the private sectors. In Uganda, whereas the private sector was trained on the new malaria treatment policy, the subsidized medicines procured with GFATM funds and later from the local manufacturer were distributed to the public and private not for profit sectors only. The private-for-profit facilities and pharmacies had to procure the medicines from the open market at a cost almost 10 times the cost of the one in the public sector. This resulted to limited access to ACTs in the private sector due to cost, yet, use of the private health sector in Uganda is significant with $59 \%$ of first consultations taking place in the private sector [45]. The Affordable Medicines Facility for malaria aims at providing more subsidized ACTs to both public and private sectors and will potentially assist to address this gap. Private-public partnerships should be put into consideration in designing policy changes.

\section{Implementation}

Changing a policy based on evidence does not automatically guarantee its implementation. There are operational issues which may relate to the health system in general but also to health workers' attitudes that need to be addressed. In the case of Uganda, evaluation studies showed variations in the new drug policy uptake by the health workers, ranging from $34 \%$ to $64 \%$. Evidence shows that reasons for this are several; the lack adequate supplies of AL, concern about AL costs, and the availability of non-recommended antimalarials like Amodiaquine caused prescription confusion [46]. Health-system-related challenges faced in Uganda included weak stock management and the resultant stockouts; health workers also had difficulties quantifying needs for the different packs when making orders. Supervision was not undertaken regularly, and diagnostic capacity was weak even when the new guidance on parasitological diagnosis before prescription of ACTs was issued. Available stocks of nonrecommended antimalarials were significant. Whereas this may have been due to the high level of stock-outs of AL in most sites, this to some extent undermined implementation of the new policy where AL was available. Kangwana et al. noted that the key benchmark of successful drug policy implementation is ensuring availability of recommended medicines at service delivery levels [47].

Although there was good progress in implementation of the policy, more supervision needed to be done to assure health workers of the availability of ACTs and ensure they prescribe ACTs for all uncomplicated malaria, especially when ACTs were available at the health facilities. The need to review the importance of color coding was also flagged as an issue requiring further scrutiny. Improving availability of AL, strengthening the supply chain, and diagnostic capacity were also noted as key to successful implementation of the new drug policy.

Among the remedial efforts to counter rampant stockouts in Uganda was starting local manufacturing of ACTs. Similarly, in Sudan, a year into implementation of the drug policy change 4 local manufacturing companies had registered their ACT products in Sudan. The extent to which this can address ACT shortages depends on several factors. In the case of Uganda improvements were modest given the low production capacity of the local firm in its infancy stage and low government expenditure on medicines; it was supposed to purchase ACTs using own resources. Per capita expenditure on medicines was only US\$ 1.7 in 2006/07 far below an estimated requirement of US\$ 7.5 [28]. In the case of Sudan, the cost of the drug was prohibitive to an ordinary Sudanese given the fact that services in public facilities were not free and, indeed, a household survey showed that treatment with ACTs was only $10.5 \%$ on average and cost was a major hindrance [30].

\section{Conclusion and Lessons Learnt}

Uganda adopted ACT treatment policy for malaria in 2005 at a time when the CQ/SP combination was evidently not the best treatment option for malaria cases in Uganda. Policy change was facilitated by the availability of funds from GFATM. The planning for policy change was meticulously done; however the implementation has been plagued by rampant stock-outs of the ACTs. Until the ACTs are available at the service delivery centres at health facility and community levels, the full impact of the new policy change may not be achieved, and the use of non-recommended antimalarials will remain a problem. The advent of AMFm and the local manufacturing of ACTs provide opportunities for ensuring unlimited access to ACTs by all but factors that may hinder realization of this objective need to be addressed.

In malaria endemic countries, drug policy change is something to be expected at one point in time. Countries should consider institutionalizing necessary processes, engagement of all stakeholders, constant dialogue using systematically collected data but must also agree on what kind of data is crucial. Comprehensive cost estimates should be computed to undertake all key activities and funding mobilized for successful policy change processes and implementation. The private sector remains a significant source of health care for the population, and improving access to new drug polices will involve strong partnerships, regulation and addressing impediments to accessing care in the private sector as much as feasible.

\section{Acknowledgments}

The authors would like to acknowledge Dr. J. B. Rwakimari, the then Programme Manager of NMCP for his contribution and stewardship in leading the drug policy change process 
and all members of the task force and its subcommittees for their contributions in planning for and participating in the rolling out of the new drug policy. Thanks also go to different researchers whose findings contributed to the evidence that informed the policy change and those who conducted studies to review the level of implementation of the new policy. Their gratitude is also extended to WHO experts particularly Dr. Peter Olumese from Geneva and Dr. Josephine Nambooze from Harare that provided technical guidance throughout the policy change process and the former WHO Representatives of Uganda, Dr. Oladapo Walker and Dr. Melville George, who provided technical support during the drug policy change process, during implementation, and in addressing the different challenges that were being faced during the implementation. Special thanks go to the Government of Uganda and GFATM for providing funding for the drug policy change activities and procurement of ACTs.

\section{References}

[1] P. E. Okello, W. Van Bortel, A. M. Byaruhanga et al., "Variation in malaria transmission intensity in seven sites throughout Uganda," American Journal of Tropical Medicine and Hygiene, vol. 75, no. 2, pp. 219-225, 2006.

[2] Roll Back Malaria Partnership, "Global Malaria Action Plan," 2008, http://www.rollbackmalaria.org/gmap/0-5.html.

[3] Ministry of Health, "Uganda Malaria Control Policy," 2000.

[4] M. R. Kamya, N. N. Bakyaita, A. O. Talisuna, W. M. Were, and S. G. Staedke, "Increasing antimalarial drug resistance in Uganda and revision of the national drug policy," Tropical Medicine and International Health, vol. 7, no. 12, pp. 10311041, 2002.

[5] World Health Organization, Regional Office for Africa, Brazzaville, "Framework for Developing, Implementing, and Updating Antimalaria Treatment Policy: A Guide for Country Malaria Control Programmes," 2003, http://afrolib.afro.who .int/documents/2003/english/framedrugp.pdf.

[6] World Health Organization, "Global report on antimalarial drug efficacy and drug resistance: 2000-2010," 2010, http: //www.searo.who.int/LinkFiles/Malaria_antimalarial_drug_efficacy10.pdf.

[7] A. O. Talisuna, A. Nalunkuma-Kazibwe, N. Bakyaita et al., "Efficacy of sulphadoxine-pyrimethamine alone or combined with amodiaquine or chloroquine for the treatment of uncomplicated falciparum malaria in Ugandan children," Tropical Medicine and International Health, vol. 9, no. 2, pp. 222-229, 2004.

[8] N. Bakyaita, G. Dorsey, A. Yeka et al., "Sulfadoxinepyrimethamine plus chloroquine or amodiaquine for uncomplicated falciparum malaria: a randomized, multisite trial to guide national policy in Uganda," American Journal of Tropical Medicine and Hygiene, vol. 72, no. 5, pp. 573-580, 2005.

[9] S. G. Staedke, A. Mpimbaza, M. R. Kamya, B. K. Nzarubara, G. Dorsey, and P. J. Rosenthal, "Combination treatments for uncomplicated falciparum malaria in Kampala, Uganda: randomised clinical trial," The Lancet, vol. 364, no. 9449, pp. 1950-1957, 2004.

[10] Ministry of Health, Uganda, "National policy on malaria treatment 2005," 2005, http://www.health.go.ug/mcp/NationalPolicyonMalariaTreatment(07_03_06).pdf.
[11] World Health Organization, "Position of WHO's Roll Back Malaria Department on malaria treatment policy," 2003, http://www.who.int/malaria/publications/atoz/who_apt_position.pdf.

[12] A. Attaran, K. I. Barnes, C. Curtis et al., "WHO, the Global Fund, and medical malpractice in malaria treatment," The Lancet, vol. 363, no. 9404, pp. 237-240, 2004.

[13] C. Fogg, F. Bajunirwe, P. Piola et al., "Adherence to a sixdose regimen of artemether-lumefantrine for treatment of uncomplicated Plasmodium falciparum malaria in Uganda," American Journal of Tropical Medicine and Hygiene, vol. 71, no. 5, pp. 525-530, 2004.

[14] S. G. Staedke, M. R. Kamya, G. Dorsey et al., "Amodiaquine, sulfadoxine/pyrimethamine, and combination therapy for treatment of uncomplicated falciparum malaria in Kampala, Uganda: a randomised trial," The Lancet, vol. 358, no. 9279, pp. 368-374, 2001.

[15] G. Dorsey, D. Njama, M. R. Kamya et al., "Sulfadoxine/ pyrimethamine alone or with amodiaquine or artesunate for treatment of uncomplicated malaria: a longitudinal randomised trial," The Lancet, vol. 360, no. 9350, pp. 2031-2038, 2002.

[16] S. Ogwang, M. Engl, M. Vigl, H. Kollaritsch, G. Wiedermann, and W. H. Wernsdorfer, "Clinical and parasitological response of Plasmodium falciparum to chloroquine and sulfadoxine/pyrimethamine in rural Uganda," Wiener Klinische Wochenschrift, vol. 115, no. 3, pp. 45-49, 2003.

[17] R. Ndyomugyenyi, P. Magnussen, and S. Clarke, "The efficacy of chloroquine, sulfadoxine-pyrimethamine and a combination of both for the treatment of uncomplicated Plasmodium falciparum malaria in an area of low transmission in western Uganda," Tropical Medicine and International Health, vol. 9, no. 1, pp. 47-52, 2004.

[18] A. F. Gasasira, G. Dorsey, B. Nzarubara et al., "Comparative efficacy of aminoquinoline-antifolate combinations for the treatment of uncomplicated falciparum malaria in Kampala, Uganda," American Journal of Tropical Medicine and Hygiene, vol. 68, no. 2, pp. 127-132, 2003.

[19] F. Checchi, P. Piola, C. Kosack et al., "Antimalarial efficacy of sulfadoxine-pyrimethamine, amodiaquine and a combination of chloroquine plus sulfadoxine-pyrimethamine in Bundi Bugyo, western Uganda," Tropical Medicine and International Health, vol. 9, no. 4, pp. 445-450, 2004.

[20] A. Yeka, K. Banek, N. Bakyaita et al., "Artemesinin versus nonartemesinin combination therapy for uncomplicated malaria: randomized clinical trials from four sites in Uganda," PLoS Medicine, vol. 2, no. 7, pp. 654-662, 2005.

[21] National Drug Authority, Uganda, "Human drug register for January 2011,” 2011, http://www.nda.or.ug/docs/Human_ list.pdf.

[22] WHO; Promoting quality of medicines programme (PQM) USAID, "Quality of antimalarials in Sub Saharan Africa," 2010.

[23] F. K. Kato, "The malaria treatment policy change process in Uganda," 2006.

[24] National Malaria Control Programme, Ministry of Health, Uganda Malaria Research Centre, and Malaria Consortium, "Assessing the availability of the first and second line antimalarials in selected health facilities in Uganda," 2007.

[25] National Malaria Control Programme, "Analysis of malaria attendances according to AL dosage age groups," 2007.

[26] D. Zurovac, J. Tibendarana, J. Nankabirwa, J. Ssekitooleko, A. Talisuna, and J. B. Rwakimari, "Evaluation of outpatient 
malaria case-management under artemether-lumefantrine treatment policy in Uganda," 2007.

[27] L. Kapiriri and D. K. Martin, "The Global Fund Secretariat's suspension of funding to Uganda: how could this have been avoided?" Bulletin of the World Health Organization, vol. 84, no. 7, pp. 576-580, 2006.

[28] Ministry of Health, "Annual health sector performance reports for the financial year 2006/07," 2007.

[29] J. A. Mulligan, R. Mandike, N. Palmer et al., "The costs of changing national policy: lessons from malaria treatment policy guidelines in Tanzania," Tropical Medicine and International Health, vol. 11, no. 4, pp. 452-461, 2006.

[30] E. M. Malik, T. A. Mohamed, K. A. Elmardi et al., "From chloroquine to artemisinin-based combination therapy: the Sudanese experience," Malaria Journal, vol. 5, article no. 65, 2006.

[31] R. Shretta, J. Omumbo, B. Rapuoda, and R. W. Snow, "Using evidence to change antimalarial drug policy in Kenya," Tropical Medicine and International Health, vol. 5, no. 11, pp. 755-764, 2000.

[32] P. Ringwald, T. Sukwa, L. K. Basco et al., "Monitoring of drugresistant malaria in Africa," The Lancet, vol. 360, no. 9336, pp. 875-876, 2002.

[33] A. W. Holly, D. Durrheim, and R. Shretta, "The process of changing national malaria treatment policy: lessons from country-level studies," Health Policy and Planning, vol. 19, no. 6, pp. 356-370, 2004.

[34] G. M. Mubyazi and M. A. Gonzalez-Block, "Research influence on antimalarial drug policy change in Tanzania: case study of replacing chloroquine with sulfadoxine-pyrimethamine as the first-line drug," Malaria Journal, vol. 4, article number 51, 2005.

[35] H. A. Williams, D. Durrheim, and R. Shretta, "The process of changing national malaria treatment policy: lessons from country-level studies," Health Policy and Planning, vol. 19, no. 6, pp. 356-370, 2004.

[36] M. Jowett, N. Miller, and N. Mnzava, "Malaria expenditure analysis. Tanzania case study. Report prepared for DFID-EA (Tanzania) and the Roll Back Malaria Initiative," International Programme, Centre for Health Economics, University of York, York, UK, 2000.

[37] C. A. Goodman, P. G. Coleman, and A. J. Mills, "Changing the first line drug for malaria treatment-Cost-effectiveness analysis with highly uncertain inter-temporal trade-offs," Health Economics, vol. 10, no. 8, pp. 731-749, 2001.

[38] W. K. Asenso-Okyere and J. A. Dzator, "Household cost of seeking malaria care. A retrospective study of two districts in Ghana," Social Science and Medicine, vol. 45, no. 5, pp. 659667, 1997.

[39] J. O. Nabyonga, F. Ssengooba, and S. Okuonzi, "Can donor aid for health be effective in a poor country? Assessment of prerequisites for aid effectiveness in Uganda," The Pan African Medical Journal, vol. 3, p. 9, 2009.

[40] N. Oomman and M. Bernstein, Following the Funding for HIV/AIDS: A Comparative Analysis of the Funding Practices of PEPFAR, the Global Fund and World Bank MAP in Mozambique, Uganda and Zambia, Center for Global Development, Washington, Wash, USA, 2007.

[41] Kaiser Daily HIV/AIDS report, "HIV/AIDS/global financial crisis could harm HIV/AIDS funding," Piot says, 2008, http: //www.global healthreporting.org/article.asp?DR_ID=55258.
[42] S. A. Okuonzi and J. Macrae, "Whose policy is it anyway? International and national influences on health policy development in Uganda," Health Policy and Planning, vol. 10, no. 2, pp. 122-132, 1995.

[43] S. Abdulla, C. Goodman, P. Coleman, G. Mubyazi, N. Kikumbih, and T. Okorosobo, "The Costs, Effects and CostEffectiveness of Changing the First-Line Drug for the Treatment of Malaria in Tanzania," Health Economics and Financing Programme (HEFP), LSHTM. Working Paper No. 01/00, 2000 http://www.dfid.gov.uk/r4d/PDF/Outputs/HealthEcFin KP/WP01_00.pdf.

[44] J. A. Mulligan, R. Mandike, N. Palmer et al., "The costs of changing national policy: lessons from malaria treatment policy guidelines in Tanzania," Tropical Medicine and International Health, vol. 11, no. 4, pp. 452-461, 2006.

[45] Uganda Bureau of Statistics, "National household Survey report," 2005.

[46] B. Wasunna, D. Zurovac, C. A. Goodman, and R. W. Snow, "Why don't health workers prescribe ACT? A qualitative study of factors affecting the prescription of artemetherlumefantrine," Malaria Journal, vol. 7, article no. 29, 2008.

[47] B. B. Kangwana, J. Njogu, B. Wasunna et al., "Short report: malaria drug shortages in kenya: a major failure to provide access to effective treatment," American Journal of Tropical Medicine and Hygiene, vol. 80, no. 5, pp. 737-738, 2009. 


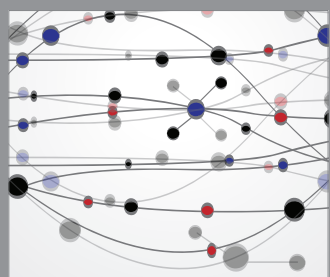

The Scientific World Journal
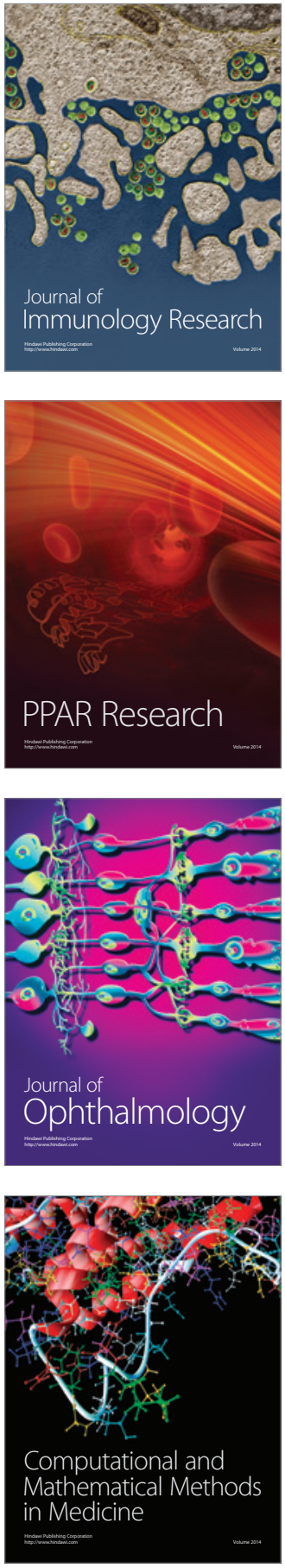

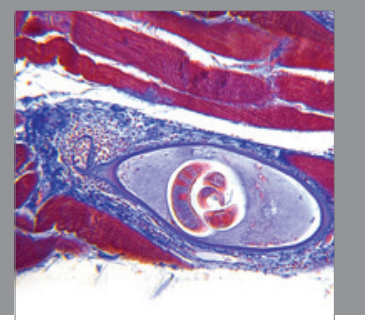

Gastroenterology

Research and Practice
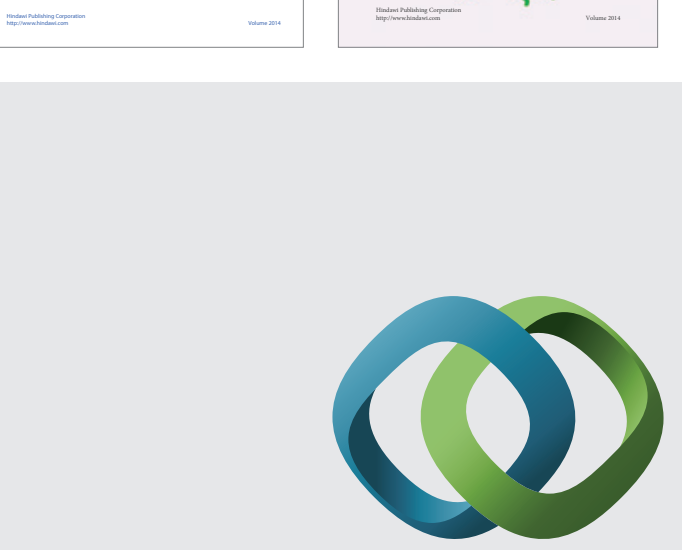

\section{Hindawi}

Submit your manuscripts at

http://www.hindawi.com
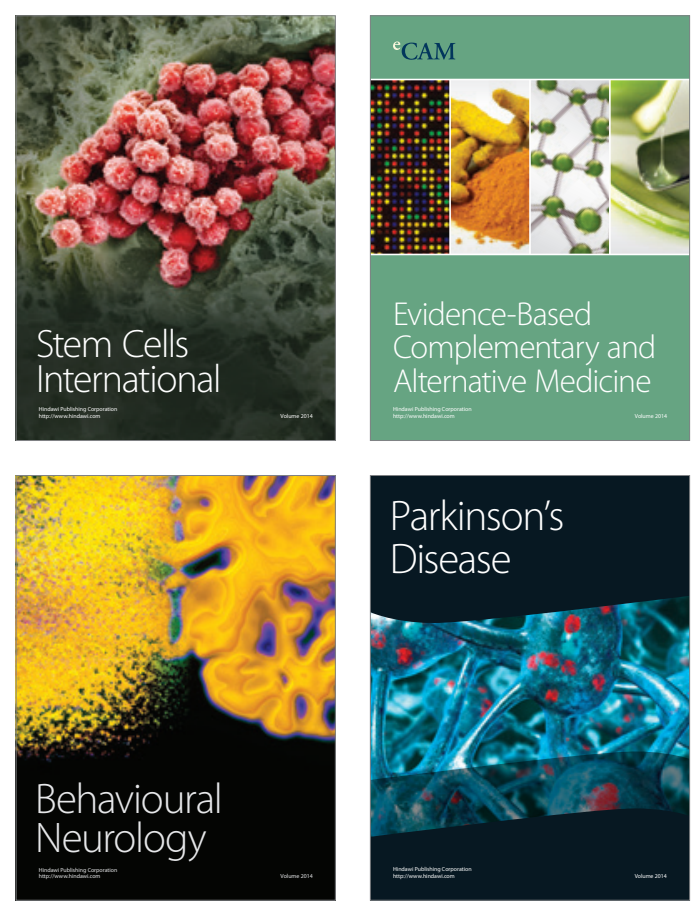

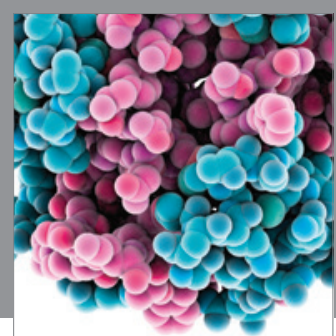

Journal of
Diabetes Research

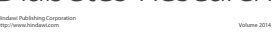

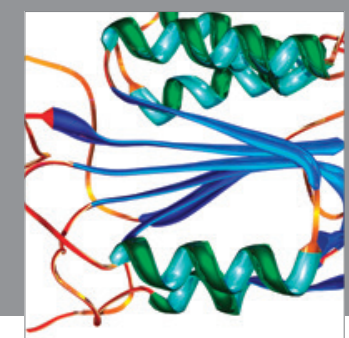

Disease Markers
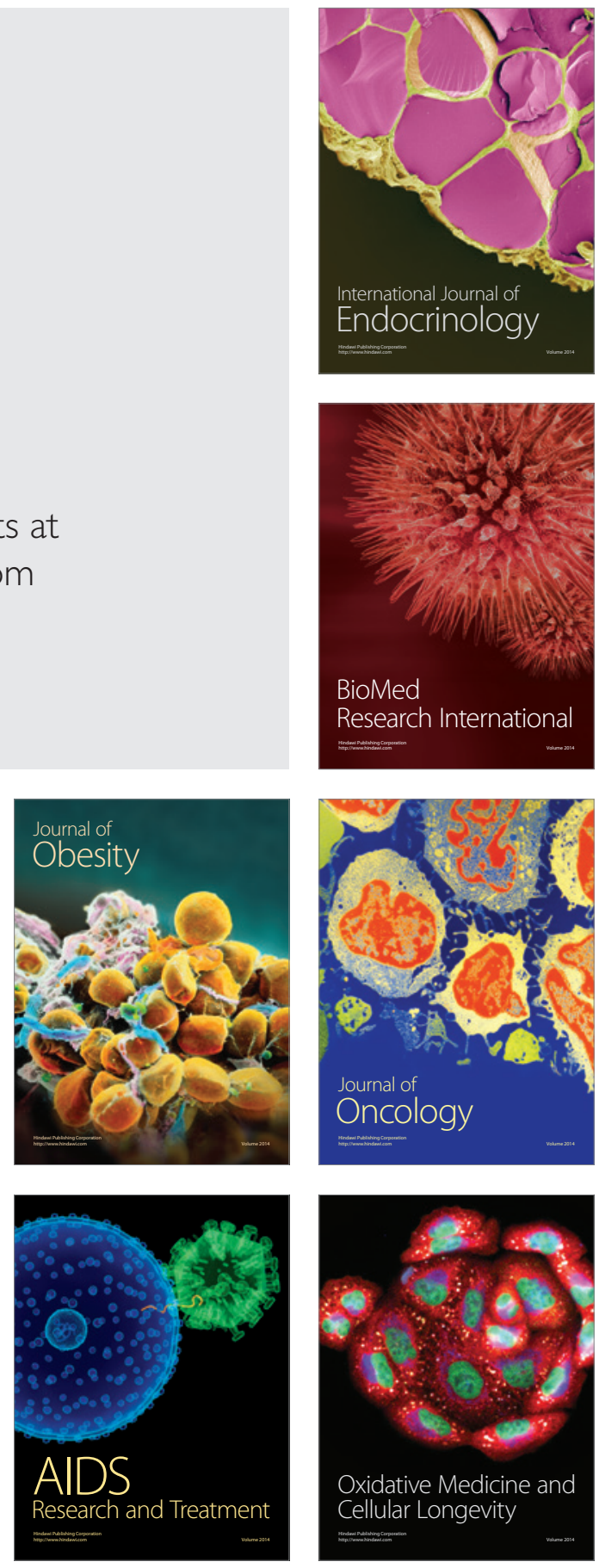